\section{Self-contained tissue factories}

\section{By Tracey Baas, Senior Editor}

Cell therapy for liver disease typically involves delivery of hepatocytes to the liver intraportal vein, which carries a risk of hemorrhage, elevated portal pressure and portal vein thrombosis. Now, a University of Pittsburgh School of Medicine team thinks it has found a better delivery route-the lymph nodes. The team has mouse data demonstrating that transplantation of cells into lymph nodes led to generation of ectopic tissue that exhibited liver function, ${ }^{1}$ and the group is testing the approach in pig models of liver failure.

The Pittsburgh team previously showed that intraperitoneal injection of hepatocytes rescued mice from liver failure if the transplanted cells migrated to and colonized nearby lymph nodes. ${ }^{2}$ The result made sense, as the lymph nodes are highly vascularized, provide direct access to essential nutrients and growth factors found in the blood, and contain cells that secrete chemokines that enhance cell recruitment and can sustain growth of diverse tissue types.

For the new study, the team opted to directly inject hepatocytes into jejunal lymph nodes located within the peritoneal cavity. In a mouse model of lethal metabolic liver failure, transplantation of syngeneic hepatocytes led to engraftment in the nodes, persistence for more than 25 weeks and rescue of the animals from liver failure. The findings were reproduced using popliteal, axillary and periportal lymph nodes, showing that multiple nodes can support engraftment and organ rescue.

In mice, the researchers generated the equivalent of about $70 \%$ of normal liver mass in one lymph node. About $30 \%$ of normal liver mass provides minimum hepatic function in a human. ${ }^{3}$

Mice also were rescued from liver failure with allogeneic hepatocyte transfer in combination with immunosuppressive agents to prevent rejection.

The researchers also saw positive results in models of pancreatic and thymic function.

In a mouse model of diabetes, islets transplanted into the lymph nodes engrafted, produced C-peptide and glucagon and restored blood glucose levels to those of normal mice within six weeks. One mouse showed normal blood glucose levels at six months post-transplant.

In athymic nude mice, transplantation of minced thymus tissue to lymph nodes led to engraftment and the presence of $\mathrm{T}$ cells in the peripheral blood for at least 10 months. Those $\mathrm{T}$ cells mounted proper responses against tumor cell transplants.

Together, the findings provide the first example that lymph nodes may be a useful ectopic site for functional cellular transplant to restore organ function.

Results were published in Nature Biotechnology.

"The lymph node is an intriguing location for cell therapy, since metastatic cancer cells grow here and now we find injected healthy liver cells can also grow in lymph nodes," said Sanjeev Gupta, professor of gastroenterology and liver diseases and chair of translational medicine at the Albert Einstein College of Medicine of Yeshiva University. "While the mouse studies do show some aspect of liver function in transplanted cells and rescue of diseased animals, we need answers to more questions about the level of liver functions that may be supported in lymph nodes. For instance,
"While the mouse studies do show some aspect of liver function in transplanted cells and rescue of diseased animals, we need answers to more questions about the level of liver functions that may be supported in lymph nodes."

-Sanjeev Gupta,

Albert Einstein College of Medicine of Yeshiva University would transplanted cells in lymph nodes reproduce all types of functions present in the liver, and if so, will those functions be at adequate levels and persist indefinitely?"

"I look forward to further characterization of tissue functions, especially in additional models of liver failure, including drug-induced acute liver toxicity with impaired liver regeneration. But the current findings are exciting and provide the basis for developing this new concept," added Gupta.

Johannes Zakrzewski noted that "the technique may be useful in situations when the liver is failing slowly, and animal models should be picked appropriately." Zakrzewski is a pediatric oncologist and a researcher who specializes in cell therapy and bone marrow transplantation at Memorial Sloan-Kettering Cancer Center.

"Important next steps for the team should be a GMP-grade system for obtaining the cells and using larger, more clinically relevant animal models, such as swine or primate, for scaled-up transplantation and long-term follow up to characterize engraftment and function of the transplants," continued Zakrzewski. "Mice are not the best for longterm studies because they have life spans of about two years. Because liver transplants are often performed on individuals 50 years or older, it might also be interesting to attempt transplantation in aged large animals."

Tim Bertram, president of R\&D and CSO at Tengion Inc., had a balanced view about the new method. "On the one hand, the lymph node does provide a potentially more practical, minimally invasive site for cell delivery" than intraportal vein injection, he said. "It also provides an ideal location to produce biological molecules that can be transported by the lymph circulation system to other areas of the body where they would be active by escaping immediate liver metabolism. On the other hand, the results are less exciting to me if they can't induce an immunological benefit, such as tolerance from the lymph node injection, and still need to use immunosuppression." 
Tengion's Neo-Urinary Conduit is in Phase I testing. The product is made from a combination of a patient's own cells and other materials to allow urine flow from the kidneys to outside the body after radical cystectomy in patients with bladder cancer.

Zakrzewski said irradiation of the patient's thymus could potentially avoid the need for immunosuppressants. Once the patient's thymus is weakened, thymus tissue from the hepatocyte donor could first be transplanted to one set of lymph nodes to provide an immune system tolerant to the donor hepatocytes. The hepatocytes could then be transplanted to another set of lymph nodes to provide liver function.

\section{Going to the pigs}

"Our first ongoing studies are focusing on transplanting hepatocytes to the lymph nodes in pig models of surgically induced liver injury," said Eric Lagasse, leader of the Pittsburgh group. "We want to see if we can rescue organ function in larger animals that will require greater numbers of transplanted cells than those used in mice. We showed that mice could be rescued by modifying one lymph node. For pigs, we will be determining how many lymph nodes will have to be modified to generate enough ectopic organ mass for rescue."

"Yes, we could work with older pigs, but there is no evidence that liver regeneration fails in older recipients," Lagasse continued. "I don't foresee regeneration issues in patients older than 50; patients older than 80 might be more relevant."

Lagasse is associate professor of pathology and director of the Cancer Stem Cell Center at the McGowan Institute for Regenerative Medicine at the University of Pittsburgh School of Medicine.

The human body contains about 500-600 lymph nodes, so "we don't foresee manipulating multiple nodes to present a problem," he said. "Cancer patients that have had lymph nodes removed during surgery for their cancer have functional immune systems."
He added, "We're going to be realistic by first focusing on allogeneic transplant using hepatocytes obtained from organs, which will require immunosuppression. But the far-reaching hope is that in the future hepatocytes will be obtained from induced pluripotent stem cells derived from patient fibroblasts, thus circumventing the need for suppression."

"For now, we have experiments in progress to investigate the concept mentioned [by Zakrzewski]," Lagasse told SciBX. "We are working to demonstrate that induced tolerance is possible by transplanting thymus plus cells or transplanting thymus plus an organ from the same donor. This should provide a way to circumvent the need for immunosuppressants in the near future."

University of Pittsburgh School of Medicine has filed for a patent covering the findings. The IP is available for licensing.

Baas, T. SciBX 5(41); doi:10.1038/scibx.2012.1074

Published online Oct. 18, 2012

\section{REFERENCES}

1. Komori, J. et al. Nat. Biotechnol.; published online Sept. 23, 2012; doi:10.1038/nbt.2379

Contact: Eric Lagasse, University of Pittsburgh School of Medicine, Pittsburgh, $\mathrm{Pa}$.

e-mail: lagasse@pitt.edu

2. Hoppo, T. et al. Gastroenterology 140, 656-666 (2011)

3. Lo, C.-M. et al. Transplantation 68, 1112-1116 (1999)

\section{COMPANIES AND INSTITUTIONS MENTIONED}

Albert Einstein College of Medicine of Yeshiva University, New York, N.Y.

McGowan Institute for Regenerative Medicine, Pittsburgh, Pa. Memorial Sloan-Kettering Cancer Center, New York, N.Y.

Tengion Inc. (OTCQB:TNGN), Winston-Salem, N.C.

University of Pittsburgh School of Medicine, Pittsburgh, Pa. 